\title{
Role of non-coding RNA in pancreatic cancer (Review)
}

\author{
YINGHAO $\mathrm{LV}^{*}$ and SHUAI HUANG* \\ Department of Hepatobiliary and Pancreatic Surgery, The First Affiliated Hospital of Zhengzhou University, \\ Zhengzhou University, Zhengzhou, Henan 450052, P.R. China
}

Received November 29, 2018; Accepted June 5, 2019

DOI: $10.3892 / \mathrm{ol} .2019 .10758$

\begin{abstract}
Pancreatic cancer is a malignant disease that develops rapidly and carries a poor prognosis.Currently, surgery is the only radical treatment. Non-coding RNAs (ncRNAs) are protein-free RNAs produced by genome transcription; they play important roles in regulating gene expression, participating in epigenetic modification, cell proliferation, differentiation and reproduction. ncRNAs also play key roles in the development of cancer; microRNA (miRNA) and long non-coding RNA (lncRNA) may lead the way to new treatments for pancreatic cancer. miRNAs are short-chain ncRNAs (19-24 nt) that inhibit the degradation of protein translation or their target gene mRNAs to regulate gene expression. IncRNAs contain > $200 \mathrm{nt}$ of ncRNA and play important regulatory roles in a number of malignant tumors, in terms of tumor cell proliferation, apoptosis, invasion and distant metastasis. IncRNAs can be exploited for the diagnosis and treatment of pancreatic cancer and have substantial prospects for clinical application. Nevertheless, the molecular mechanism of their regulation and function, as well as the significance of other ncRNAs, such as piwi-interacting RNA, in the pathogenesis of pancreatic cancer, are largely unknown. In this review, the structures of ncRNAs with various classifications, as well as the functions and important roles of ncRNAs in the diagnosis and treatment of pancreatic cancer are reviewed.
\end{abstract}

\section{Contents}

1. Introduction

2. miRNA

3. piRNA

4. IncRNA

Correspondence to: Dr Shuai Huang, Department of Hepatobiliary and Pancreatic Surgery, The First Affiliated Hospital of Zhengzhou University, Zhengzhou University, 1 Longhu Middle Ring Road, Zhengzhou, Henan 450052, P.R. China

E-mail: fcchuangs@zzu.edu.cn

${ }^{*}$ Contributed equally

Key words: non-coding RNA, cancer, tumorigenesis, metastasis, diagnosis, future therapeutics, treatment
5. circRNA

6. Conclusions

\section{Introduction}

Non-coding RNAs (ncRNAs) are protein-free RNAs produced by genome transcription. There are 20,000-25,000 genes encoded in the human genome, of which $40-90 \%$ are estimated to be regulated by miRNA (1). A variety of RNAs, including microRNAs (miRNAs/miRs) and long non-coding RNAs (lncRNAs), regulate gene expression at the transcription, post-transcription and epigenetic levels. Research has shown that ncRNA inhibits the translation activity of mRNA, regulates the expression of specific target genes, and ultimately regulates biological signaling pathways and manipulates cell fates $(2,3)$. In recent years, substantial progress has been made in basic research regarding the underlying disease mechanisms, using model organisms and new experimental systems. For example, mir-21 was shown to promote cell migration and invasion, and play a key role in cancer cell infiltration and remote metastasis. These studies have provided evidence that ncRNAs play important roles in the development and progression of pancreatic cancer $(1,4)$.

Pancreatic cancer ranks 4th in the world among all malignant tumors, with high invasiveness, early metastasis, lack of specific symptoms and high mortality. The median survival time is only 3-6 months, with a 5-year survival rate of $<5 \%$. In China, the incidence of pancreatic cancer has increased 6-fold in the past 20 years (5). In 2012, the incidence of pancreatic cancer was 4.8/100,000, ranking 7 th among all malignant tumors. Even when early diagnosed, small pancreatic cancer ( $<2 \mathrm{~cm}$ in diameter) can easily metastasize and lead quickly to the death of the patient. Surgical resection remains the only treatment for radical treatment. Nevertheless, only $15-20 \%$ of pancreatic cancer patients are suitable for surgical treatment $(1,6,7)$. Even with multidisciplinary comprehensive treatment, the median survival time of patients with advanced pancreatic cancer is only $\sim 6$ months. Furthermore, early postoperative recurrence and occult metastasis also reduce the efficacy of surgical treatment. Even after systemic therapy, liver metastasis occurs in $50 \%$ of patients. However, the main reasons for the high mortality rates of pancreatic cancer are the failure of an early diagnosis, before it moves to other organs, and the resistance of the cancer to existing therapies (8).

In recent years, with the development of molecular biology, especially gene sequencing technology, the mechanism 
of action of lncRNAs in various types of cancer has been gradually revealed, and the study of ncRNAs has been significantly augmented for various types of cancer. In the case of pancreatic cancer, various IncRNAs are involved in the biological behavior and signal pathways of metastatic pancreatic cancer, including the cell cycle, apoptosis, autophagy, epithelial-mesenchymal transition (EMT) and cancer stem cells (CSCs) $(6,9)$. The present review focuses on the characteristics and biological roles of several ncRNAs, with particular emphasis on their role in pancreatic cancer (Table I).

\section{2. miRNA}

miRNA is the most characteristic ncRNA; it is a short sequence of RNA with a length of $\sim 22 \mathrm{nt}$. miRNA expression is related to the type of tumor, as well as its occurrence and development (10). Therefore, miRNA, as a potential tumor marker, has been the focus of clinical research. As miRNA binds mRNA through incomplete pairing, thereby inhibiting translation, the same miRNA can simultaneously regulate several target genes and signaling pathways (11). Furthermore, miRNAs are also conservative, testable and can exist stably outside cells. Therefore, they have become diagnostic markers and therapeutic targets of tumors (8).

miRNAs are usually produced as primary transcripts for RNA polymerase 2 transcription, known as pri-miRNAs. Recent studies have shown that miRNAs can act as intermediates, regulating a large part of human gene transcription (12). At present, feedback loops are widely known to exist between miRNAs and basic transcription factors (TFs). Although there are numerous differences between them, TFs positively or negatively regulate the expression of miRNA, while miRNAs inhibit the translation of TF-miRNA (13). Studies have shown that, after transfection of let-7 into pancreatic cancer cells, the proliferation capacity of the transfected cells is significantly inhibited, presumably related to the effect of miRNA on the expression of the k-ras gene and the activation of mitogen-activated protein kinase $(8,14)$.

There are various ways in which miRNAs alter the levels of gene expression. In particular, they typically bind to the mRNA 3'UTR. Genomic DNA is transcribed into mRNA, and mRNA has 5'UTR and 3'UTR structures. miRNA binds to the mRNA 3'UTR, and then alters the translation process after mRNA, thereby affecting the expression results of the particular gene (Fig. 1) (15-18).

Generation of model organisms and their use in miRNA functional studies. Despite the fact that Caenorhabditis elegans do not develop cancer, they have been widely used as model organisms to identify molecular functions and to describe pathways involved in normal cellular processes that are severely impaired in cancer, including cell proliferation, differentiation, metabolism and death (19). Full sequencing of C. elegans genomes showed that $\sim 60 \%$ of miRNAs have a human homologous gene. As the number of conserved miRNAs in the miRNA family of $C$. elegans decreased, the study of miRNA function in C. elegans eliminated redundancy as a barrier to be overcome. Moreover, C. elegans are self-fertilized hermaphrodites that can produce large numbers of genetically identical offspring $(19,20)$. The visual tracking of C. elegans, with a well-organized transparent body, makes for an excellent model system. Phenotypic and genetic screening, the application of molecular techniques, and the development of transgenic C. elegans have identified a number of key miRNAs, and have elucidated their mechanisms of action. Research on C. elegans has also contributed to the understanding of the molecular basis of the miRNA biological genome. For example, Dicer C. elegans ortholog gene, dcr-1, involved in RNA-mediated silencing, has been identified as a key component necessary for the processing of mature let-7 from its precursor molecule $(19,21)$.

The zebrafish is a model system that plays an important role in elucidating the functional role of miRNA in both normal and cancer cells. Since let-7 was identified during in silico prediction as a cross-species conserved miRNA, the other miRNAs, initially found in zebrafish, have considerable homology with the miRNAs encoded by humans and other vertebrates, in terms of composition and function $(20,21)$. Thanks to a complete angiogenesis and immune system, as well as a developed organ system, human cancer cells were successfully transplanted into zebrafish embryos, enabling the tumor to grow in the host microenvironment. Therefore, the zebrafish was also shown to be a successful model system for assessing tumor response to anticancer therapy in vivo. In summary, despite the fact that the zebrafish is a simple model system, the striking properties of D. rerio permit the use of this organism to better understand the effects of aberrant changes in endogenous levels of certain miRNAs $(21,22)$. The zebrafish has been proven to be a successful model system, leading to the recognition of several miRNAs through basic biochemical and molecular studies, and has great potential as a model to identify clinically relevant miRNAs.

Previous studies used mir-520d-5p to confirm its possible therapeutic effect on cancer cells $(23,24)$. The size and signal intensity of green fluorescent protein (GFP)-expressing tumors was measured in mice weekly, for 4 weeks after subcutaneous inoculation. It was found that, compared with the control group, 520d treatment, which consisted of introducing exogenous gene $520 \mathrm{~d}$ into mice, inhibited tumor growth by $>80 \%$ per week, resulting in the disappearance of $\sim 30 \%$ of the tumors. In mice with tumor disappearance, the presence of human genomic material at the injection site was quantitatively detected by alu-PCR, confirming the coexistence of the two cell sources. GFP was expressed in connective tissue at each site where the immune-deficient mouse tumors disappeared, and $\sim 0.1 \%$ of the extracted DNA contained human genomic material.

miRNAs can be divided into two forms, namely those with high and low expression in pancreatic cancer cells, similar to proto-oncogenes and tumor suppressor genes. These two factors have wide influence on biological pathways, and their abnormal regulation is an important factor leading to the development of pancreatic cancer (25).

Oncogenic miRNAs. Researchers detected 95 miRNAs with abnormal expression in pancreatic cancer using RT-PCR. Among these, mir-196a, mir-190, mir-186, mir-221, mir-222, mir-200b, mir-15b and mir-95 were significantly upregulated, while let-7, mir-96, mir-15 and mir-132 were significantly downregulated (2). Studies reported that mir-217 
Table I. Specific types and functions of different ncRNAs and their association with pancreatic cancer.

\begin{tabular}{|c|c|c|c|c|}
\hline First author (year) & Type of RNAs & Length, nt & Function & (Refs.) \\
\hline $\begin{array}{l}\mathrm{Fu}(2017), \text { Gao (2018) } \\
\text { Gao (2018) }\end{array}$ & Small nucleolar RNAs & $70-200$ & Involved in maturation of other ncRNAs & $(6,7,59)$ \\
\hline $\mathrm{Fu}(2017)$, Gao (2018) & Small nuclear RNAs & $100-215$ & $\begin{array}{l}\text { Binding to proteins to form splicers that } \\
\text { control selective splicing }\end{array}$ & $(6,59)$ \\
\hline $\mathrm{Gu}(2017)$ & Small interfering RNAs & $21-22$ & $\begin{array}{l}\text { Silencing specific genes in a sequence-specific } \\
\text { manner }\end{array}$ & $(60)$ \\
\hline Gao (2018), Gu (2017) & Small ncRNAs & $<200$ & Carrying amino acids that bind to the ribosome & $(59,60)$ \\
\hline Peng (2016) & lncRNAs & $>200$ & Component of ribosomes & $(61)$ \\
\hline Peng (2016), Peng (2016) & $\begin{array}{l}\text { Ribosomal 28S and } \\
\text { 18SRNA }\end{array}$ & $200-5,050$ & Component of ribosomes & $(2,61)$ \\
\hline Vorvis (2016), Wang (2017) & Piwi-interacting RNA & $25-34$ & $\begin{array}{l}\text { Controlling retro transposition and regulating } \\
\text { methylation }\end{array}$ & $(3,71)$ \\
\hline Xiong (2017), Zhang (2017) & $\begin{array}{l}\text { Promoter-associated } \\
\text { short RNAs }\end{array}$ & $<200$ & $\begin{array}{l}\text { Regulating gene expression through interaction } \\
\text { with gene promoter sites }\end{array}$ & $(11,63)$ \\
\hline Zhou (2017) & $\begin{array}{l}\text { Long intergenic ncRNAs } \\
\text { or long intronic ncRNAs }\end{array}$ & $>200$ & Various & $(68)$ \\
\hline Zhou (2017) & Antisense RNA & $>200$ & Binding and blocking of mRNA target genes & $(68)$ \\
\hline Zhou (2017), Zhang (2017) & $\begin{array}{l}\text { Promoter-associated } \\
\text { short RNAs }\end{array}$ & $>200$ & $\begin{array}{l}\text { Regulating gene expression through interaction } \\
\text { with gene promoter sites }\end{array}$ & $(31,85)$ \\
\hline Zhang (2017) & $\begin{array}{l}\text { Telomere-associated } \\
\text { ncRNAs }\end{array}$ & $\begin{array}{l}100 \mathrm{bp} \\
>9 \mathrm{~kb}\end{array}$ & Negative telomere regulators & (79) \\
\hline Zhang (2017), Zhang (2017) & $\begin{array}{l}\text { Transcribed } \\
\text { ultra-conserved regions }\end{array}$ & $200-799$ & $\begin{array}{l}\text { Long-range enhancer-like activity, maintenance } \\
\text { of splicing factor expression levels and } \\
\text { transcription regulation }\end{array}$ & $(79,85)$ \\
\hline
\end{tabular}

ncRNAs, non-coding RNAs; lncRNAs, long ncRNAs.

and mir-148a/b were downregulated in pancreatic cancer cells, mir-155 and mir-21 were found to be highly expressed in breast cancer and they may also be used as landmarks of pancreatic cancer $(10,18)$. Previous studies analyzed tumors, including those of the lung, stomach, prostate, rectum and pancreas, and found that mir-17-5p, mir-20a, mir-21, mir-92, mir-106a and mir-155 were all highly expressed in tumor cells $(10,18)$. Of these, mir-20a was highly sensitive and specific, and could be used as a marker for differentiating various stages of pancreatic cancer from paracarcinoma tissue and chronic pancreatitis. Previous studies compared the plasma levels of 50 pancreatic cancer patients and 10 healthy patients, and found that 37 types of miRNA were decreased and 54 types of miRNA were increased $(2,26,27)$. Among these miRNAs, mir-21 is an oncogene. Therefore, it is thought that the high expression of mir-21 can be used as an indicator of poor survival in pancreatic cancer patients, as well as a prognostic marker. Additionally, the miRNA expression profile, including mir-99, mir-100 and mir-100-1/2, varied in pancreatic cancer tissues, adjacent and chronic pancreatitis tissues (CP) $(27,28)$. mir-199a-1 and mir-199a-2 were relatively highly expressed in pancreatic cancer and $\mathrm{CP}$, possibly indicating that they stimulate the growth of neoplasms. This could be caused by the interference of inflammatory factors. The study also found that mir-196a-2 was significantly correlated with patient survival. Other studies found that miRNA precursors with increased expression were mir-22-1, -424, -301,-100, -376a, -125b-1, -021, -016-1, -181a, c, -092-1, -092-1, -212, -107, -024-1, 2, 1et-7f-1, 1et-7d, mir-345, -142-p, -139 (14,29). These results were similar to those of Volinia (30). Volinia (30) and Zhou et al (31) used endpoint immuno PCR to detect the expression of mir-196a in normal pancreatic cells, pancreatic duct epithelial neoplasms (PanIN, precancerous) cells and pancreatic cancer cells. It was found that, in normal pancreatic cells, mir-196a expression was not present; however, PanIN and all tumor cells showed positive expression of miR-196a, suggesting that mir-196a might indicate the carcinogenic transformation process of pancreatic cells. Volinia (30) and Yu et al (29) compared the differences in expression of miRNAs in the peripheral blood of ovarian cancer patients and normal individuals, and found that mir-21, -92, -93, -126 and -29a were highly expressed in ovarian cancer. Xiong et al (11) and Yan et al (32) found that mir-184 was highly expressed in the peripheral blood of tongue squamous cell carcinoma. Yan et al (33) found that mir-92 was highly expressed in the peripheral blood of rectal cancer patients. 


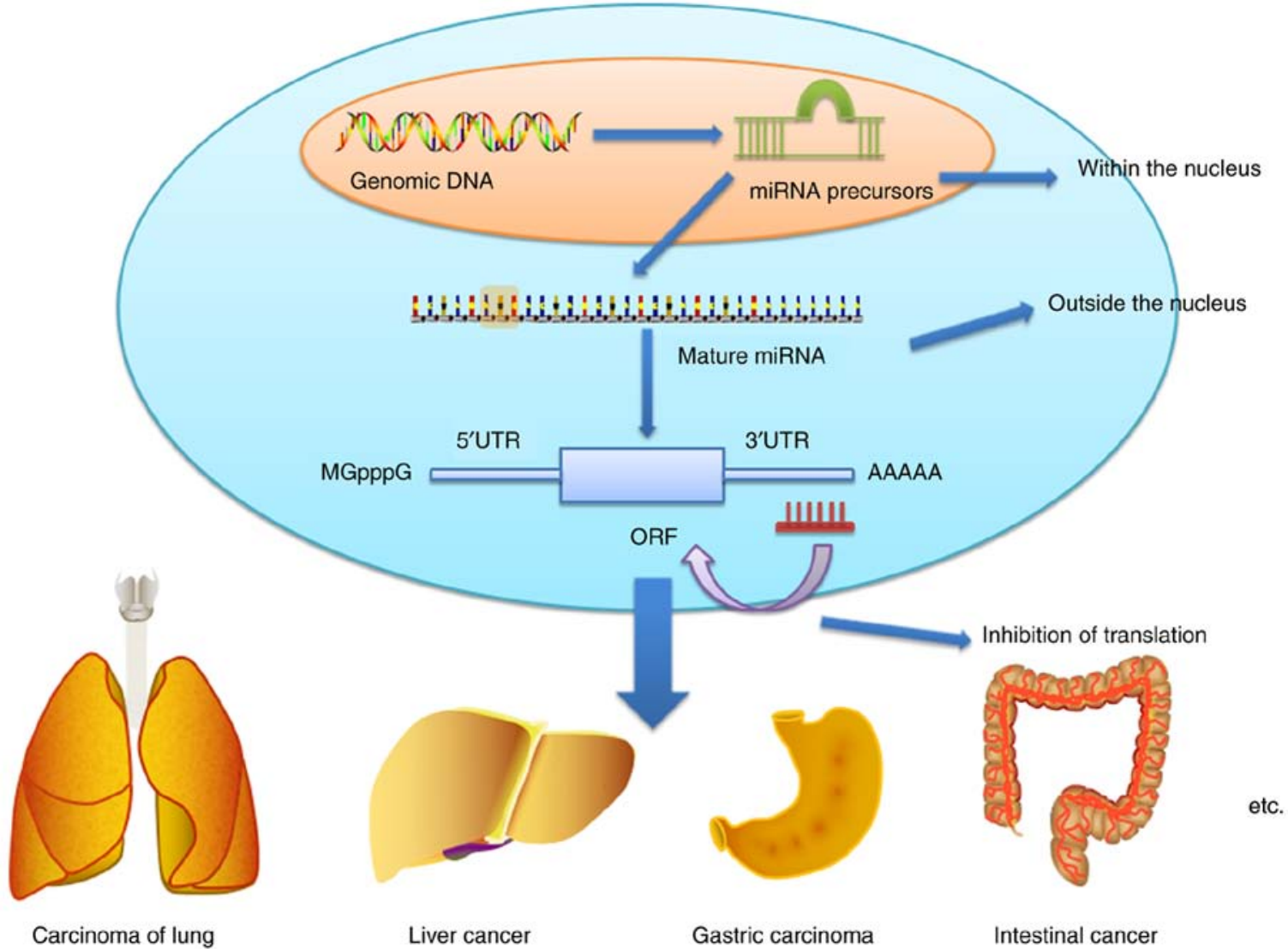

Figure 1. Mechanism by which miRNA alters gene expression, via inhibition of translation of mRNA by binding the 3'UTR. miRNA, microRNA.

Tumor suppressor gene-related miRNAs. As opposed to proto-oncogenes, some miRNAs are less well expressed in pancreatic tissue than in normal pancreatic tissue; they inhibit the development of pancreatic cancer by negatively regulating the cell cycle and proliferation. The decreased levels of mature miRNAs may be caused by defects in any step of miRNA biosynthesis, eventually leading to the expression of inappropriate miRNA target proteins. Wang et al (34) found that the expression of two miRNAs, mir-15a and mir-16a, was downregulated or missing in most patients with chronic lymphocytic leukemia (CLL). Sun et al (27) and Vassaux et al (35) found that the expression of mir-127 in some human primary tumors was downregulated or missing, while it was normal in normal cells; therefore, mir-127 was hypothesized to be a potential tumor suppressor gene. Recently, a study reported that mir-615-5p was significantly downregulated in pancreatic cancer compared with its expression in normal adjacent tissues, and that it inhibited proliferation, migration and invasion of pancreatic cancer cells by targeting AKT2 (36). The let-7 family miRNAs are also well known among all types of miRNA. mir-1181 was found to have low expression in the middle of pancreatic cancer, and it was shown to directly inhibit the expression of sox 2 and stat 3 (37). mir193-b is a tumor suppressor gene that demonstrates interaction inhibition with MillHG $(38,39)$. mir-193b negatively regulates mir31HG by directly targeting two miRNA binding sites in the MillHG sequence and inhibits proliferation of pancreatic cancer cells, while mir31HG can be used as an endogenous 'sponge' to reduce its anticancer effect $(10,40)$.
Studies on miRNAs in the treatment of pancreatic cancer have been ongoing. The Panpane-1, LPc111 and LPc006 cell lines of pancreatic cancer showed increased tolerance to gemcitabine when they were transfected with precursor mir-21; apoptosis levels decreased or proliferation levels increased. By contrast, mir-21 expression in the suit- 2 pancreatic cancer cell line was inhibited; apoptosis levels increased and proliferation activity decreased (41). Li et al (42) and Hancock and Skalsky (43) showed that mir-21 can be used as an indicator of resistance of pancreatic cancer cells in the Hengtian region. Pancreatic cancer cell lines with low expression of mir-21 are highly sensitive to fluorouracil. Li et al (18) and Hancock and Skalsky (43) screened and verified pancreatic cancer tissues and two types of pancreatic cancer cell lines that were resistant to gemcitabine using miRNA microarray. It was found that cells with high expression of mir-142-5p and mir-204 had significantly increased tolerance to gemcitabine. It was proposed that the expression levels of mir-142-5p can be thought of as indicators of resistance of pancreatic cancer cells to gemcitabine.

DNA methylation is a form of DNA chemical modification that alters the genetic expression without changing the DNA sequence, causing changes in chromatin structure, DNA conformation, DNA stability and the way DNA interacts with proteins, thereby controlling gene expression. DNA methylation has a definite effect on cell carcinogenesis in various ways. A methyl group attaches to the DNA region near the tumor suppressor gene, inhibits the activity of the gene, and effectively 'silences' the gene. Recently, researchers focused 
on a class of ncRNAs that specifically affect CCAAT enhancer binding protein $\alpha$ (CEBPA), a tumor suppressor gene $(44,45)$. CEBPA silencing is associated with acute myeloid leukemia, lung and pancreatic cancer, as well as other types of cancer. ncRNA binds DNA methyltransferase 1 to prevent methylation of CEBPA. This principle, which is likely to be applied to thousands of other genes, holds promise for 'reactivating' downregulated cancer suppressors. Researchers (46) found that the newly-discovered ncRNA specifically inhibits DNA methylation, and the introduction of RNA selectively demethylates certain genes, thereby initiating the expression of tumor suppressors. This mechanism is expected to be exploited in the treatment of a variety of diseases, including cancer (Fig. 2) $(1,8,46)$.

miRNA-301. mir-301 is highly expressed only in pancreatic cancer, suggesting that mir-301 may be a molecular marker of specificity in pancreatic cancer. Studies have shown that mir-301 is highly expressed in genes related to the invasion and metastasis of pancreatic cancer, including cox-2 and mmp-2. mir-301 is closely related to the invasion and metastasis of pancreatic cancer (47). mir-301 inhibitors effectively suppress the expression of the invasion- and metastasis-related genes cox-2 and mmp-2, and significantly inhibit the invasion and metastasis of pancreatic cancer cells, suggesting that mir-301 is effective $(10,12)$. mir-301 may be a new molecular target for the therapy of pancreatic cancer. The establishment of effective detection methods and targeted treatment strategies for mir-301 will provide important theoretical and experimental bases for the early diagnosis, treatment option selection, efficacy evaluation and prognosis in pancreatic cancer (47).

miRNA-20a. miRNA-20a was shown to be expressed at low levels in pancreatic tissue and cell lines. The expression of the miRNA inhibits the growth and invasion of pancreatic cancer cell lines, and its role in pancreatic cancer is achieved through the negative regulation of oncogenes Stat 3 and cyclin D1 (48). Studies have reported that miRNA-20a directly inhibits E2F TF1 (E2F1) and regulates the cell cycle. The miRNA also plays the role of an oncogene as it is overexpressed in a number of malignant tumors, including lung cancer and B-cell lymphoma. The mechanism involved may be the downregulation of Stat 3 and cyclin D1 expression by miRNA-20a $(16,49)$.

mir-200 family. Peng et al (2) and Qian et al (38) found that the expression of the mir-200 family is downregulated or is missing in the course of EMT. The results were related to EMT, and it was reported that EMT is closely related to the relatively low survival rate after surgical resection of pancreatic duct cell carcinoma. Studies found that the gemcitabine resistance of pancreatic cancer cells was associated with the lower expression of mir-200 and let-7 in these cells. The epithelial phenotype was restored by transfection of mir-200 with generate gemcitabine resistant cells; therefore, the epithelial marker E-calcium mucins was increased, and the leaf-cutting marker E-box zinc-finger protein was combined with vimentin to inhibit EMT and invasion of tumor cells $(27,50)$.

Therapeutic application of miRNAs. miRNA can be involved in treatment in two ways, namely the reduction and replacement of miRNA. Downregulation of miRNA is targeted at the acquisition of functions, and oncogenic miRNA can be inhibited in the following ways: i) Small molecule inhibitors act on the regulation of miRNA expression at the transcriptional level; ii) antisense oligonucleotides bind to complementary miRNAs, inducing duplex formation or miRNA degradation; iii) miRNA masking uses molecules complementary to the 3'UTR of the target miRNA to competitively inhibit downstream target effects; and iv) miRNA sponge, constructed by oligonucleotide with multiple complementary miRNA binding sites, binds to target miRNA $(51,52)$. The second strategy, miRNA substitution, is targeted at functional loss, including the restoration of functional loss by introducing systemic miRNA (miRNA mimics) or inserting miRNA-coding genes into viral construction, and reintroducing tumor suppressor miRNA $(51,53)$. Despite the promise of both approaches, two major problems have, thus far, hampered the development and effectiveness of miRNA-based therapies. The first is that it is necessary to obtain tissue-specific delivery and develop more efficient cellular uptake of synthetic oligonucleotides to achieve constant target inhibition. The second important problem is the relatively low efficiency of miRNAs in body fluids or tissues, seeking to restore lost function or block tumor-enhanced miRNAs, which have been partially overcome by the construction of locked nucleic acids (LNA). The most successful use of the so-called 'LNA anti-miRs' resulted in the first miRNA-based clinical trial for the treatment of hepatitis $\mathrm{C}$ virus infection, which used lna-anti-mir to target mir-122 (54,55).

Chemotherapy is an important treatment strategy for most pancreatic cancer patients. Chen Q and Hong L reported the results of a phase III clinical trial that directly compared gemcitabine with 5-FU and showed that gemcitabine significantly improved the median survival time. Soon thereafter, gemcitabine became the first-line and gold-standard chemotherapy for pancreatic cancer $(51,54)$. Nevertheless, drug resistance to gemcitabine and other drugs may lead to treatment failure. Despite the fact that research in drug resistance has been ongoing, the mechanism of its occurrence has not been fully explained. The three most common reasons for acquiring drug resistance are the expression of energy-demanding transporters, insensitivity to drug-induced apoptosis and drug detoxification-induced mechanisms $(53,55)$. In one study, oncogenic mir-155 levels were shown to increase after gemcitabine was used for the treatment of pancreatic cancer cells. mir-200a, mir-200b and mir-200c were downregulated in gemcitabine-resistant pancreatic cancer cells (55). A recent report showed that mir-34 was involved in the self-renewal of pancreatic CSCs, and the deletion of mir-34 in pancreatic cancer was related to the enrichment of CSCs, which are not sensitive to chemotherapy $(52,56)$. There is also evidence that miRNAs may regulate EMT by regulating cadherin-1 and other molecules, mediating various cell resistance mechanisms $(54,56)$. When studying the expression levels of mir-200 and let-7 in gemcitabine-resistant pancreatic cancer cells with the EMT phenotype, the downregulated expression of mir-200 family upregulated cadherin-1 and downregulated ZeB1 and vimentin (EMT inducer). Downregulation of ZeB1 in mesenchymal Panc1 cells led to upregulation of mir-200c (56). 


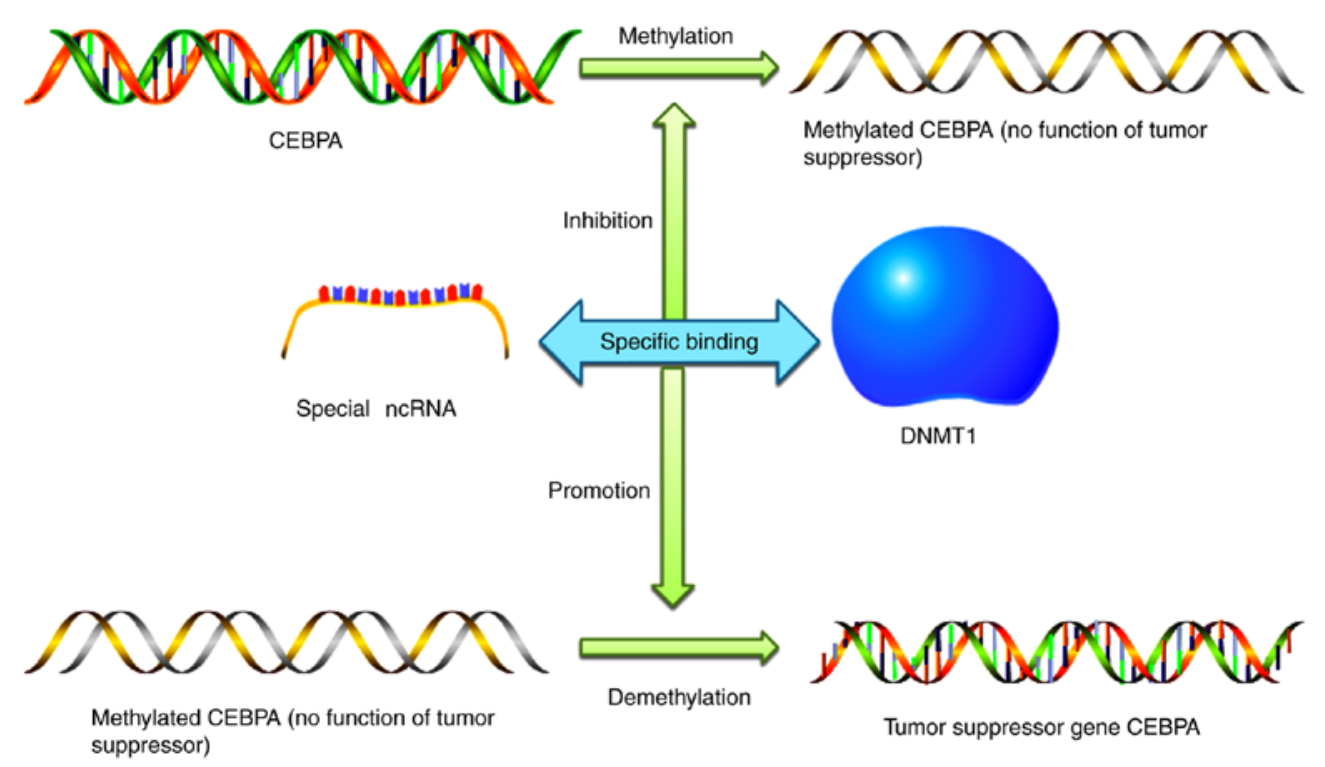

Figure 2. ncRNA influences the methylation of DNA by binding DNMT1, thereby inhibiting the inactivation of tumor suppressor gene CEBPA. ncRNA, non-coding RNA; DNMT1, DNA methyltransferase 1; CEBPA, CCAAT enhancer binding protein $\alpha$.

Therefore, by targeting CSCs or EMT phenotype cells that lead to tumor recurrence and metastasis, the re-expression of specific miRNAs can be used as a new strategy for the treatment of pancreatic cancer.

\section{PIRNA}

piRNA research began more recently. piRNAs are ncRNAs of 24-30 nt in length. piRNAs rely on the piwi protein group that inhibits the expression of transposable elements in two ways by forming a complex with piwi. At present, there are two proposed pathways for generating piRNAs, namely a primary processing pathway and a 'ping-pong' amplification loop. Similar to miRNA, the 5 '-end also has a strong uracil bias $(57,58)$. A previous study has shown that piRNA exists in mammalian germ cells, and regulates the gene silencing pathway by binding with the piwi subfamily proteins to form piRNA complexes (2). The discovery of piRNA opens a new field for the study of non-coding small molecular RNAs. To date, piRNA research has achieved interim results. However, it is too early to predict their use in biomedicine $(2,58)$. At present, piRNA regulates mRNA primarily via two mechanisms: Degradation and shear. CAF1 mRNA in miRNA regulation has a very important role in degradation. Studies in mouse CAF1 by Andersen et al (57) demonstrated its combination with piRNA/piwi complexes. This complex process recognizes the target mRNA after removal of adenosine piRNA, eventually causing mRNA degradation. Repoila et al (47) reported the piRNA/piwi-mediated shear mechanism of mRNA. There remain numerous problems that require further discussion $(44,58)$. For example, the homologous conservation of piRNA sequences in various species is not easy to analyze. In piRNA panspermia, it remains unknown how the division mechanisms of the 3'-end and the piRNA amplification cycle are regulated. piRNA research not only enriches the research content of small molecular RNA, but also helps further the understanding of molecular regulation and mechanisms of biological gametes with substantial theoretical value (57). There are three members of the piwi subfamily, namely MIWI, MILI and MIWI2, which are specific proteins in germ cells and closely related to the occurrence of sperm. Knockout of MIWI, MILI or MIWI2 resulted in sperm production disorders in male mice (58). MILI was expressed during early spermatogenesis, from mitosis of spermatocytes to the pachytene stage of spermatocytes, and the MILI deficient mice stopped at pachytene stage. MIWI is expressed later, from pachytene to spheric spermatogenesis. Knockout of MIWI causes spermatogenesis to stop at spheric spermatogenesis. However, mice with knockout MIWI2 had defects in meiosis progression at early meiosis stage and significant loss of germ cells with age (57).

piRNA forms a complex with piwi protein that is involved in the regulation of target sequences. Piwi is also detected in cancer cells. There are four piwi proteins in humans: PIWIL1/HIWI, PIWIL2/HILI, PIWIL3 and PIWIL4/HIWI2, and there are specific types of piwi proteins in various cancers (57). Piwi proteins play four major roles in cancer cells: The promotion of cell proliferation, the inhibition of apoptosis, the enhancement cell migration and the maintenance of genomic stability. Cancer cells have unlimited ability to proliferate and migrate, and they also have the ability to escape apoptosis and divide continuously $(57,58)$. In order to maintain this ability, the relative stability of their genome structure must be ensured. Although piwi protein was found in cancer cells, piRNA that binds to piwi protein was not found, suggesting that a number of unknown functions of the piwi and piRNA complex remain undiscovered.

\section{IncRNA}

lncRNA is a long-chain ncRNA of $>200$ nt. Studies have shown that abnormal expression of IncRNA is closely related to the development of various tumors and can show the function of oncogenes and tumor suppressor genes $(59,60)$. 
According to the currently known lncRNAs, the following functions can be summarized as follows: i) Some lncRNAs bind epigenetic-related proteins after transcription, modifying specific sequences, activating or inhibiting the activity of chromatin-related regions, and regulating cell activities, such as X-chromosome inactivation; ii) lncRNAs inhibit or promote gene transcription via base pairing and recruitment of TFs; iii) IncRNA are precursors to the formation of miRNAs and regulate the network regulation of miRNA by mediating the generation of miRNAs; and iv) IncRNAs participate in the formation of nuclear sub-structures by regulating related genes $(5,9)$. Furthermore, lncRNAs can be involved in gene network regulation in three ways: i) In the transcriptional level: When lncRNA itself is transcribed, wherein it interferes with the transcription of adjacent genes, and where it can be used as an endogenous competitive RNA to control myoblast differentiation; ii) in the post-transcriptional level: By interacting with miRNA, lncRNA affects post-transcriptional translation of related mRNA, thereby promoting the malignant transformation and proliferation of cells; and iii) in the epigenetic level: IncRNA regulates histone modification to silence expression of adjacent genes, also affecting chromosome reconstruction to promote tumor invasion and metastasis (42). Nevertheless, the mechanism of action of lncRNA on tumorigenesis and development remains in its infancy. Further research and elaboration of the mechanism of action of lncRNA will provide new approaches to tumor diagnosis, treatment and prognosis.

In terms of structure, IncRNAs usually have the same structure as mRNAs, including 5'-caps and 3'-polyadenylated tails, and they are spliced to form the final product. Despite the fact that IncRNAs are poorly sequentially conserved, some studies suggest that their functions may be relatively conserved $(3,61)$. Furthermore, genes are usually located very close to lncRNA in the genome, and according to the proximity of their protein-coding genes, IncRNA can be divided into five categories: i) Antisense lncRNA; ii) intronic transcript lncRNA; iii) large intergenic lncRNA; iv) promoter-associated lncRNA; and v) UTR-associated lncRNA $(42,61)$. Similar to miRNA, lncRNA plays both carcinogenic and anti-oncogene roles in terms of occurrence, development and metastasis of various tumors. While similarities between lncRNA and miRNA exist, they are usually highly diverse in structure and function. HOTAIR was the first lncRNA shown to regulate gene expression in the form of reverse transcription, as identified by Vorvis et al (3) IncRNA HOTAIR showed higher expression in pancreatic cancer with peripheral tissue infiltration and lymph node metastasis $(29,62)$. Survival analysis of pancreatic cancer patients showed that patients with low expression of lncRNA HOTAIR had a significantly longer survival time than those with high expression, which is of great significance in predicting prognosis (62).

Oncogenic lncRNA. To date, a partial oncogenic lncRNA has been identified. Zhang et al (63) used gene chip technology and found that ZEB1 antisense RNA 1 (zeb1-as1) was upregulated in liver cancer tissues, especially in metastatic tumor tissues. zeb1-as1 overexpression was associated with abnormal DNA methylation. Yan et al (33) and Volinia et al (64) found that colorectal cancer-associated lncRNA showed high expression in colorectal cancer tissues. A recent study showed that repulsive guidance molecule B as1 (rgmb-as1), upregulated in non-small cell lung cancer, was associated with the degree of tumor differentiation, lymph node metastasis and clinical stage, and was negatively correlated with RGMB (25). As a pro-apoptotic factor, GDFl5 inhibits the proliferation of cancer cells, and HOTAIR binding with the GDFl5 gene region weakens its anticancer effect in pancreatic cancer tissues.

Tumor suppressor gene-related lncRNA. In contrast to oncogenic lncRNA, some lncRNAs have been shown to act as tumor suppressors. Ferreira and Esteller (16) and He et al (36) found that the expression of cell adhesion molecule as1 (cadm1-as1) was downregulated in renal clear cell carcinoma, and that interfering with the expression of cadm1-as1 promoted the growth and migration of tumor cells, and reduced the apoptosis rate. Some researchers found that the low expression of taurine upregulated gene 1 (TUG1) was related to high-level TNM stage, large tumor size and lower overall survival in patients with non-small cell lung cancer. The inhibition of TUG1 expression significantly promoted the proliferation of tumor cells and tissues $(40,65)$. One study found that overexpression of tslc1-as1 induced upregulation of TSLC1 and inhibited proliferation, migration and invasion of tumor cells. The expression of tslc1-as1 was positively correlated with other tumor suppressor genes, including neurofibromatosis type 1, von Hippel-Lindau tumor suppressor and phosphoinositide-3-kinase regulatory subunit 1 , while it was negatively correlated with the oncogene B-Raf proto-oncogene serine/threonine kinase. In an in vitro study, Li et al (42) and Yu et al (29) found that the overexpression of growth arrest-specific 5 (GAS5) significantly inhibited the proliferation and invasion of PANC1 and bxpc-3 cell lines. After inhibition of GAS5 expression by RNA interference, GAS5 was arrested in the $\mathrm{G}_{0} / \mathrm{G}_{1}$ stage. The proportion of cells in the stage decreased, and more cells were in the $\mathrm{S}$ stage, suggesting that GAS5 regulates the cell cycle of pancreatic cancer cells and inhibits the invasion of cancer cells $(66,67)$.

$\mathrm{Bc}-819$ is a plasmid vector capable of expressing diphtheria toxin A chain under the regulation of $\mathrm{H} 19$, which has antitumor effects. Bc-819 was initially used in the treatment of bladder cancer. In human trials, after ingestion, it expresses a large amount of diphtheria toxin in cells, causing tumor volume reduction. With the extensive development of clinical trials, the effective concentration and dose of bc- 819 have been further determined $(33,64)$. On this basis, Zhang et al $(63)$ and Zhou et al (68) used gemcitabine, a first-line chemotherapy drug for pancreatic cancer, in combination with bc-819 in an animal model of pancreatic cancer, and achieved good efficacy. Pvt1 oncogene (PVT1) is directly related to the sensitivity of pancreatic cancer to gemcitabine. In the human pancreatic cancer aspc-1 cell line, decreased PVT1 activity improved the sensitivity to gemcitabine. When Kim et al (69) conducted high-throughput sequencing for pancreatic cancer, it was found that homeobox A distal transcript antisense RNA (HOTTIP) increased the expression in pancreatic cancer and was involved in the development of resistance to gemcitabine through HOXA13. Tseng et al (50) found that PVT1 in the aspc-1 cell line regulated the sensitivity of cells to gemcitabine, and showed that the overexpression of full-length PVT1 positive-sense cDNA increased the sensitivity of cells to 
gemcitabine. Meller et al (40) confirmed that malat-1 reduces the sensitivity of aspc-1 and cfpac-1 cell lines to gemcitabine chemotherapy.

lncRNA mir3lHG. IncRNA mir31HG is one of the lncRNA molecules with the most significant differential expression, primarily located in the cytoplasm. Recent studies have shown that mir-31 is located in the intron region of mir31HG, and the transcription of mir-31 is regulated by mir31HG. Both miR-31 and mir31HG were found to be significantly positively correlated in pancreatic cancer tissues, while the expression of mir31HG was not changed with the decrease of mir-31 level, suggesting that both may exert their respective roles independently $(33,64)$. mir-31 expression did not change at low concentrations of mir31HG, and the inhibition or expression of mir-31 did not affect the expression of mir31HG. At the transcriptional level, mir31HG and mir-31 did not regulate the expression of each other, and their biological functions were independent. The pancreatic cancer gene function of mir31HG acted by promoting the proliferation, migration and invasion of pancreatic cancer cells $(34,64)$. Another study suggested that the low expression of mir-193b in pancreatic cancer has a significant negative correlation with the expression of mir31HG (70). mir-193b was found to negatively regulate the expression and function of mir31HG by directly targeting mir31HG.

Maternally expressed 3 (MEG3). MEG3 is an imprinted gene expressed by the mother, located in the positive $\mathrm{q} 32$ region of chromosome 14, and its transcription product MEG3 is a 1,595-bp long RNA molecule. Another study found that tumor protein p53 and growth differentiation factor 15 play important roles in the regulation of MEG3 (71). There are two differentially methylated regions (DMRs) upstream of the MEG3 gene: IG-DMR and meg3-dmr. Previous studies found that these two DMRs may be the control centers of gene expression for the entire dlk1-meg3 unit (61,71). Zhang et al (63) found that the meg3-dmr was fully methylated in a neuroblastoma cell line. The ultimate effect was an increase in the methylation level of the MEG3 gene promoter, which was also the direct cause of MEG3 inactivation. Zhang et al (63) and Peng and Jiang (61) found that the expression of MEG3 in pancreatic cancer was negatively correlated with the clinicopathological features of the tumor. MEG3 overexpression inhibited pancreatic cancer activity by regulating the PI3K/AKT/bcl-2/BAX/cyclin D1/p53 and PI3K/AKT/mmp-2/mmp-9 signaling pathways. AKT, bcl1-2 cell cycle protein and D1 are found in normal tissues and inhibit pyruvate dehydrogenase kinase during cell division to promote the expression of p53 and BAX. They inhibit the invasion and migration of the pancreatic cancer PANC1 cell line by inhibiting the expression of mmp-2 and mmp-9 $(39,72)$.

H19 imprinted maternally expressed transcript (H19). H19 plays an oncogenic role by antagonizing hmga2-mediated EMT through antagonizing the anti-oncogene let-7, which is primarily located in the cytoplasm and has a high expression level during embryonic development, while it is strongly suppressed after birth (70). As the first exon of H19 transcription, mir-675 is negatively correlated with the expression level of H19. As a key TF, E2F-1 is positively correlated with the expression levels of H19, and E2F-1 is a direct target of miR675. mir-675 reduces $\mathrm{H} 19$ activation by affecting the expression of E2F-1 protein by binding to the 3'UTR on E2F-1 mRNA (73). Furthermore, some studies have shown that $\mathrm{H} 19$ has the dual function of oncogene and tumor suppressor. H19 was the earliest lncRNA used for pancreatic cancer treatment. DNA treatment controlled by the H19 gene sequence, whether used alone or combined with gemcitabine, significantly improves the therapeutic effect in pancreatic cancer $(36,74,75)$.

PVT1. PVT1 has the potential to cause cancer in numerous malignant tumors. However, only recently, studies revealed the role of PVT1 in pancreatic cancer. Tseng et al (50) found that the expression levels of PVT1 were positively correlated with clinical stage and total survival time in pancreatic cancer. PVT1 is a regulator of the sensitivity of pancreatic cancer cells to gemcitabine. Other studies showed that curcumin inhibited the prc2-pvtl-c-myc axis by inhibiting the prc2-subunit enhancer of zeste 2 polycomb repressive complex 2 subunit, targeting CSCs and enhancing the sensitivity of cancer cells to chemical therapeutic agents $(2,42)$. Tutar (39) and Tseng et al (50) found that PVT1 promoted the proliferation and metastasis of pancreatic cancer cells by acting as a 'molecular sponge' to inhibit mir-448 activation, thus inhibiting the effect of SERPINE1 mRNA binding protein 1 on target cells. Nevertheless, studies on the detailed mechanism of PVT1 in pancreatic cancer and other tumor types are rare. Furthermore, other genes play a crucial role in pancreatic cancer, and they are located at various sites (11) (Table II).

Drug resistance and sensitivity in drug application. The clinical efficacy of some pancreatic cancer-targeted drugs, such as gemcitabine, remains controversial. To date, some lncRNAs have been found to be associated with pancreatic cancer therapy. Sethi et al (76) and Taucher et al (25) used a high-throughput microarray to measure the expression profiles of IncRNAs in pancreatic cancer tissue and found that quantitative PCR upregulated the expression of HOTTIP in pancreatic cancer. Moreover, the study also reported that HOTTIP promoted gemcitabine resistance through HOXA13, suggesting that HOTTIP and HOXA13 may be new therapeutic targets for pancreatic cancer. Unfortunately, the study failed to identify the downstream signaling pathways that HOTTIP regulates (77). Tang et al (78) found that the PVT1 gene regulated the sensitivity of gemcitabine in the human aspc-1 pancreatic cancer cell line. It was found that that the sensitivity of gemcitabine increased when the full-length PVT1 cDNA was overexpressed from the antisense strand, and decreased when the full-length PVT1 cDNA was overexpressed from the positive-sense strand. Nevertheless, the underlying mechanism by which PVT1 regulates gemcitabine in pancreatic tumorigenesis remains unknown. Xiong et al (11) found that malat-1 reduced the chemical sensitivity of gemcitabine in aspc-1 and cfpac-1 cells.

\section{5. circRNA}

Circular RNAs (circRNAs) are a class of ncRNAs involved in the regulation of transcription and post-transcriptional gene expression; they are also a class of covalently bonded 
Table II. Function and location of typical genes involved in pancreatic cancer.

\begin{tabular}{|c|c|c|c|c|}
\hline First author (year) & Gene name & Genomic localization & Involvement in pancreatic cancer & (Refs.) \\
\hline Wang (2017), Liu (2016) & H19 & $11 \mathrm{p} 15.5$ & $\begin{array}{l}\text { Cell proliferation, invasion, targeted } \\
\text { therapy }\end{array}$ & $(70,73)$ \\
\hline Martens-Uzunova (2014) & PVT 1 & $8 \mathrm{q} 24.21$ & Drug resistance & (65) \\
\hline Vallot (2017), Xie (2017) & HULC & $6 \mathrm{p} 24.3$ & Cell proliferation & $(66,67)$ \\
\hline Meller (2015) & AF 339813 & $13 \mathrm{q} 31.3$ & Cell proliferation and apoptosis & (40) \\
\hline Pang (2015) & Gas5 & $1 \mathrm{q} 25.1$ & Cell proliferation, the cell cycle & $(72)$ \\
\hline Kim (2013) & HOTAIR & $12 q 12.13$ & $\begin{array}{l}\text { Proliferation, apoptosis, drug resistance, } \\
\text { chemotherapy sensitivity }\end{array}$ & (69) \\
\hline Fuschi (2019) & UCA1 & 19pl13.12 & Proliferation, migration, invasion & (75) \\
\hline $\begin{array}{l}\text { He (2018), } \\
\text { Martens-Uzunova (2014) }\end{array}$ & ROR & $1 \mathrm{p} 31.3$ & $\begin{array}{l}\text { Proliferation, migration, invasion, drug } \\
\text { resistance (autophagy) }\end{array}$ & $(36,65)$ \\
\hline Li (2017) & TUG1 & $22 \mathrm{q} 12.2$ & Proliferation, migration (EMT) & $(42)$ \\
\hline Meller (2015), Hancock (2018) & ENST00000480739 & $12 \mathrm{q} 13$ & Unspecified & $(40,43)$ \\
\hline
\end{tabular}

single-stranded ncRNAs. circRNA has the following biological characteristics: i) As opposed to linear RNA, circRNA forms a closed covalently circular structure that it is more stable than linear RNA; ii) compared with linear mRNA, the species of circRNA are more abundant and varied; iii) non-circRNA is primarily derived from exons, and a large number of circRNAs exist in the cytoplasm of eukaryotic cells; nevertheless, a small number of circRNAs, derived from introns or intron fragments, exist in the nucleus with spatiotemporal specificity of expression in various tissues at various stages of development; iv) most circRNAs are ncRNAs that are evolutionarily conserved; and v) most circRNAs are regulated at the transcriptional or post-transcriptional level $(79,80)$. Knupp (81) and Li et al (82) compared microarray expression profiles of circRNA in pancreatic ductal adenocarcinoma and normal first-line tissues, and found that the expression of circRNA was different in two different tissues, and the most typical of which were ci-7 and ci-sirt7. circRNA alters the occurrence of pancreatic cancer, and is expressed in normal and tumor tissues with specificity, being more stable than linear RNA, and at higher concentration in serum. These characteristics make circRNA an ideal biomarker of pancreatic cancer and a substantial potential therapeutic target (83-85). Nevertheless, research on the correlation between circRNA and pancreatic cancer remains in the preliminary stage, and the study of the association between circRNA and pancreatic cancer is a hotspot in the current field of pancreatic cancer research.

\section{Conclusions}

ncRNAs have become hotspots of modern medical research; they promote the proliferation and metastasis of pancreatic cancer through EMT, competitive endogenous RNA (ceRNA), the cell cycle, apoptosis and other pathways. These various aspects are isolated; nevertheless, they are interwoven with one another. Several lncRNAs have been identified as oncogenes or tumor suppressors involved in tumorigenesis. Despite the fact that protein biomarkers are widely used in cancer detection and in follow-up studies of diseases, lncRNAs have their own advantages. IncRNA itself is an effector molecule that reflects the actual level of a tumor. The correlation between lncRNA levels and specific types of tumors makes it an accurate tool for tumor diagnosis and classification $(2,85)$. The expression of lncRNA is related to tumor therapeutic response and can be used as an indicator of therapeutic effect. Combined with other prognostic methods, lncRNAs can be used as new tumor markers, facilitating the identification of patients with poor prognosis and prolonging their survival. Furthermore, lncRNA has extensive application prospects in terms of tumor treatment $(37,85)$. Currently, IncRNA has been used in the treatment of various tumors. However, most of the current studies on IncRNA focus on the differences in the expression levels, and relatively few studies have identified molecular functions. The discovery and understanding of carcinogenic effects in pancreatic cancer are also incomplete, and the details of these mechanisms remain to be elucidated. With the continuous innovation and development of research methods, lncRNAs may become potential biomarkers and therapeutic targets for pancreatic cancer $(2,35)$.

\section{Acknowledgements}

Not applicable.

\section{Funding}

The study was supported by grants from The Youth Fund of The First Affiliated Hospital of Zhengzhou University.

\section{Availability of data and materials}

The datasets used and/or analyzed during the present study are available from the corresponding author upon reasonable request. 


\section{Authors' contributions}

YL and SH contributed to the design, as well as the writing of the review. All authors read and approved the final manuscript.

\section{Ethics approval and consent to participate}

Not applicable.

\section{Patient consent for publication}

Not applicable.

\section{Competing interests}

The authors declare that they have no competing interests.

\section{References}

1. Cech TR and Steitz JA: The noncoding RNA revolution-trashing old rules to forge new ones. Cell 157: 77-94, 2014.

2. Peng JF, Zhuang YY, Huang FT and Zhang SN: Noncoding RNAs and pancreatic cancer. World J Gastroenterol 22: 801-814, 2016

3. Vorvis C, Hatziapostolou M, Mahurkar-Joshi S, Koutsioumpa M, Williams J, Donahue TR, Poultsides GA, Eibl G and Iliopoulos D Transcriptomic and CRISPR/Cas9 technologies reveal FOXA2 as a tumor suppressor gene in pancreatic cancer. Am J Physio Gastrointest Liver Physiol 310: G1124-G1137, 2016.

4. Chandra Gupta S and Nandan Tripathi Y: Potential of long non-coding RNAs in cancer patients: From biomarkers to therapeutic targets. Int J Cancer 140: 1955-1967, 2017.

5. Duguang L, Jin H, Xiaowei Q, Peng X, Xiaodong W, Zhennan L, Jianjun Q and Jie Y: The involvement of lncRNAs in the development and progression of pancreatic cancer. Cancer Biol Ther 18: 927-936, 2017.

6. Fu Z, Chen C, Zhou Q, Wang Y, Zhao Y, Zhao X, Li W, Zheng S, Ye H, Wang L, et al: LncRNA HOTTIP modulates cancer stem cell properties in human pancreatic cancer by regulating HOXA9. Cancer Lett 410: 68-81, 2017.

7. Gao H, Gong N, Ma Z, Miao X, Chen J, Cao Y and Zhang G: LncRNA ZEB2-AS1 promotes pancreatic cancer cell growth and invasion through regulating the miR-204/HMGB1 axis. Int J Bio Macromol 116: 545-551, 2018

8. Chitkara D, Mittal A and Mahato RI: miRNAs in pancreatic cancer: Therapeutic potential, delivery challenges and strategies. Adv Drug Deliv Rev 81: 34-52, 2015.

9. Chen P, Wan D, Zheng D, Zheng Q, Wu F and Zhi Q: Long non-coding RNA UCA1 promotes the tumorigenesis in pancreatic cancer. Biomed Pharmacother 83: 1220-1226, 2016.

10. Li Y and Sarkar FH: MicroRNA targeted therapeutic approach for pancreatic cancer. Int J Biol Sci 12: 326-337, 2016.

11. Xiong G, Feng M, Yang G, Zheng S, Song X, Cao Z, You L, Zheng $\mathrm{L}, \mathrm{Hu} \mathrm{Y}$, Zhang $\mathrm{T}$ and Zhao Y: The underlying mechanisms of non-coding RNAs in the chemoresistance of pancreatic cancer. Cancer Lett 397: 94-102, 2017.

12. Abreu FB, Liu X and Tsongalis GJ: miRNA analysis in pancreatic cancer: The Dartmouth experience. Clin Chem Lab Med 55 755-762, 2017.

13. Batchu RB, Gruzdyn OV, Qazi AM, Kaur J, Mahmud EM Weaver DW and Gruber SA: Enhanced phosphorylation of p53 by microRNA-26a leading to growth inhibition of pancreatic cancer. Surgery 158: 981-987, 2015.

14. Czech B and Hannon GJ: One loop to rule them all: The ping-pong cycle and piRNA-guided silencing. Trends Biochem Sci 41: 324-337, 2016.

15. Guil S and Esteller M: RNA-RNA interactions in gene regulation: The coding and noncoding players. Trends Biochem Sci 40 248-256, 2015 .

16. Ferreira HJ and Esteller M: Non-coding RNAs, epigenetics, and cancer: Tying it all together. Cancer Metastasis Rev 37: 55-73, 2018.

17. Lai X, Wang M, McElyea SD, Sherman S, House M and Korc M A microRNA signature in circulating exosomes is superior to exosomal glypican-1 levels for diagnosing pancreatic cancer. Cancer Lett 393: 86-93, 2017.
18. Li A, Yu J, Kim H, Wolfgang CL, Canto MI, Hruban RH and Goggins M: MicroRNA array analysis finds elevated serum miR-1290 accurately distinguishes patients with low-stage pancreatic cancer from healthy and disease controls. Clin Cancer Res 19: 3600-3610, 2013.

19. Chang J, Yao M, Li Y, Zhao D, Hu S, Cui X, Liu G, Shi Q, Wang Y and Yang Y: MicroRNAs for osteosarcoma in the mouse: A meta-analysis. Oncotarget 7: 85650-85674, 2016

20. Delás MJ,Sabin LR, DolzhenkoE,Knott SR, Munera MaravillaE, Jackson BT, Wild SA, Kovacevic T, Stork EM, Zhou M, et al: lncRNA requirements for mouse acute myeloid leukemia and normal differentiation. Elife 6: e25607, 2017.

21. He L, Tian DA, Li PY and He XX: Mouse models of liver cancer: Progress and recommendations. Oncotarget 6: 23306-23322, 2015 .

22. Ishihara Y, Tsuno S, Kuwamoto S, Yamashita T, Endo Y, Miura K, Miura Y, Sato T, Hasegawa J and Miura N: Tumor-suppressive effects of atelocollagen-conjugated hsa-miR-520d-5p on un-differentiated cancer cells in a mouse xenograft model. BMC Cancer 16: 415, 2016.

23. Wei WF, Han LF, Liu D, Wu LF, Chen XJ, Yi HY, Wu XG, Zhong M, Yu YH, Liang L and Wang W: Orthotopic xenograft mouse model of cervical cancer for studying the role of MicroRNA-21 in promoting lymph node metastasis. Int J Gynecol Cancer 27: 1587-1595, 2017.

24. Zhu J, Zhu W and Wu W: MicroRNAs change the landscape of cancer resistance. Methods Mol Biol 1699: 83-89, 2018.

25. Taucher V, Mangge H and Haybaeck J: Non-coding RNAs in pancreatic cancer: Challenges and opportunities for clinical application. Cell Oncol (Dordr) 39: 295-318, 2016.

26. Moriya C, Taniguchi H, Miyata K, Nishiyama N, Kataoka K and Imai K: Inhibition of PRDM14 expression in pancreatic cancer suppresses cancer stem-like properties and liver metastasis in mice. Carcinogenesis 38: 638-648, 2017.

27. Sun XJ, Liu BY, Yan S, Jiang TH, Cheng HQ, Jiang HS, Cao Y and Mao AW: MicroRNA-29a promotes pancreatic cancer growth by inhibiting tristetraprolin. Cell Physiol Biochem 37: 707-718, 2015.

28. Wang P, Zhang J, Zhang L, Zhu Z, Fan J, Chen L, Zhuang L, Luo J, Chen $\mathrm{H}$, Liu L, et al: MicroRNA 23b regulates autophagy associated with radioresistance of pancreatic cancer cells. Gastroenterology 145: 1133-1143.e12, 2013.

29. Yu X, Zheng H, Chan MT and Wu WK: HULC: An oncogenic long non-coding RNA in human cancer. J Cell Mol Med 21: 410-417, 2017.

30. Volinia S: Unexpected findings of variability in microRNAs suggest roles in human genetics. Genome Med 4: 69, 2012.

31. Zhou B, Sun C, Hu X, Zhan H, Zou H, Feng Y, Qiu F, Zhang S, $\mathrm{Wu} \mathrm{L}$ and Zhang B: MicroRNA-195 suppresses the progression of pancreatic cancer by targeting DCLK1. Cell Physiol Biochem 44: 1867-1881, 2017.

32. Yan H, Li Q, Wu J, Hu W, Jiang J, Shi L, Yang X, Zhu D, Ji M and Wu C: MiR-629 promotes human pancreatic cancer progression by targeting FOXO3. Cell Death Dis 8: e3154, 2017.

33. Yan L, Zhang J, Guo D, Ma J, Shui SF and Han XW: IL-21R functions as an oncogenic factor and is regulated by the lncRNA MALAT1/miR-125a-3p axis in gastric cancer. Int J Oncol 54: 7-16, 2019.

34. Wang W, Zhao L, Wei X, Wang L, Liu S, Yang Y, Wang F, Sun G, Zhang J, Ma Y, et al: MicroRNA-320a promotes 5-FU resistance in human pancreatic cancer cells. Sci Rep 6: 27641, 2016.

35. Vassaux G, Angelova A, Baril P, Midoux P, Rommelaere J and Cordelier P: The promise of gene therapy for pancreatic cancer. Hum Gene Ther 27: 127-133, 2016.

36. He Y, Hu H, Wang Y, Yuan H, Lu Z, Wu P, Liu D, Tian L, Yin J, Jiang K and Miao Y: ALKBH5 inhibits pancreatic cancer motility by decreasing long non-coding RNA KCNK15-AS1 methylation. Cell Physiol Biochem 48: 838-846, 2018.

37. Zhang Y, Wang J, Huang S, Zhu X, Liu J, Yang N, Song D, Wu R, Deng W, Skogerb $\varnothing$ G, et al: Systematic identification and characterization of chicken (Gallus gallus) ncRNAs. Nucleic Acids Res 37: 6562-6574, 2009.

38. Qian Y, Feng L, Wu W, Weng T, Hu C, Hong B, Wang FXC, Shen L, Wang Q, Jin X and Yao H: MicroRNA expression profiling of pancreatic cancer cell line L3.6p1 following B7-H4 knockdown. Cell Physiol Biochem 44: 494-504, 2017.

39. Tutar Y: miRNA and cancer; computational and experimental approaches. Curr Pharm Biotechnol 15: 429, 2014.

40. Meller VH, Joshi SS and Deshpande N: Modulation of chromatin by noncoding RNA. Annu Rev Genet 49: 673-695, 2015. 
41. Li M, Radvanyi L, Yin B, Li J, Chivukula R, Lin K, Lu Y, Shen J, Chang DZ, Li D, et al: Downregulation of human endogenous retrovirus type K (HERV-K) viral env RNA in pancreatic cancer cells decreases cell proliferation and tumor growth. Clin Cancer Res 23: 5892-5911, 2017.

42. Li H, Wang X, Wen C, Huo Z, Wang W, Zhan Q, Cheng D, Chen H, Deng X, Peng C and Shen B: Long noncoding RNA NORAD, a novel competing endogenous RNA, enhances the hypoxia-induced epithelial-mesenchymal transition to promote metastasis in pancreatic cancer. Mol Cancer 16: 169, 2017.

43. Hancock MH and Skalsky RL: Roles of non-coding RNAs during herpesvirus infection. Curr Top Microbiol Immunol 419: 243-280, 2018.

44. Farshidfar F, Zheng S, Gingras MC, Newton Y, Shih J, Robertson AG, Hinoue T, Hoadley KA, Gibb EA, Roszik J, et al: Integrative genomic analysis of cholangiocarcinoma identifies distinct IDH-mutant molecular profiles. Cell Rep 18: 2780-2794, 2017.

45. Kamerkar S, LeBleu VS, Sugimoto H, Yang S, Ruivo CF, Melo SA, Lee JJ and Kalluri R: Exosomes facilitate therapeutic targeting of oncogenic KRAS in pancreatic cancer. Nature 546: 498-503, 2017

46. Binenbaum Y, Na'ara S and Gil Z: Gemcitabine resistance in pancreatic ductal adenocarcinoma. Drug Resist Updat 23: 55-68, 2015.

47. Repoila F and Darfeuille F: Small regulatory non-coding RNAs in bacteria: Physiology and mechanistic aspects. Biol Cell 101: 117-131, 2009.

48. Gu DN, Jiang MJ, Mei Z, Dai JJ, Dai CY, Fang C, Huang Q and Tian L: microRNA-7 impairs autophagy-derived pools of glucose to suppress pancreatic cancer progression. Cancer Lett 400: 69-78, 2017.

49. Karamitopoulou E, Haemmig S, Baumgartner U, Schlup C and Wartenberg M: MicroRNA dysregulation in the tumor microenvironment influences the phenotype of pancreatic cancer. Mod Pathol 30: 1116-1125, 2017.

50. Tseng YY, Moriarity BS, Gong W, Akiyama R, Tiwari A Kawakami H, Ronning $\mathrm{P}$, Reuland $\mathrm{B}$, Guenther $\mathrm{K}$, Beadnell TC, et al: PVT1 dependence in cancer with MYC copy-number increase. Nature 512: 82-86, 2014.

51. Chen Q, Wang P, Fu Y, Liu X, Xu W, Wei J, Gao W, Jiang K, Wu J and Miao Y: MicroRNA-217 inhibits cell proliferation, invasion and migration by targeting Tpd5212 in human pancreatic adenocarcinoma. Oncol Rep 38: 3567-3573, 2017.

52. Gayral M, Jo S, Hanoun N, Vignolle-Vidoni A, Lulka H, Delpu Y, Meulle A, Dufresne M, Humeau M, Chalret du Rieu M, et al: MicroRNAs as emerging biomarkers and therapeutic targets for pancreatic cancer. World J Gastroenterol 20: 11199-11209, 2014

53. Hao J, Zhang S, Zhou Y, Liu C, Hu X and Shao C: MicroRNA 421 suppresses DPC4/Smad4 in pancreatic cancer. Biochem Biophys Res Commun 406: 552-557, 2011.

54. Hong L, Yang Z, Ma J and Fan D: Function of miRNA in controlling drug resistance of human cancers. Curr Drug Targets 14 $1118-1127,2013$

55. Humeau M, Torrisani J and Cordelier P: miRNA in clinical practice: Pancreatic cancer. Clin Biochem 46: 933-936, 2013.

56. Kokuryo T, Hibino S, Suzuki K, Watanabe K, Yokoyama Y, Nagino M, Senga T and Hamaguchi M: Nek2 siRNA therapy using a portal venous port-catheter system for liver metastasis in pancreatic cancer. Cancer Sci 107: 1315-1320, 2016.

57. Andersen PR, Tirian L, Vunjak M and Brennecke J: A heterochromatin-dependent transcription machinery drives piRNA expression. Nature 549: 54-59, 2017.

58. Charlotte S: piRNAs power sperm development in the adult. Biol Reprod 94: 2016

59. Gao ZQ, Wang JF, Chen DH, Ma XS, Yang W, Zhe T and Dang XW: Long non-coding RNA GAS5 antagonizes the chemoresistance of pancreatic cancer cells through down-regulation of miR-181c-5p. Biomed Pharmacother 97: 809-817, 2018.

60. Gu L, Zhang J, Shi M, Zhan Q, Shen B and Peng C: lncRNA MEG3 had anti-cancer effects to suppress pancreatic cancer activity. Biomed Pharmacother 89: 1269-1276, 2017.

61. Peng W and Jiang A: Long noncoding RNA CCDC26 as a potential predictor biomarker contributes to tumorigenesis in pancreatic cancer. Biomed Pharmacother 83: 712-717, 2016.

62. Yang SZ, Xu F, Zhou T, Zhao X, McDonald JM and Chen Y: The long non-coding RNA HOTAIR enhances pancreatic cancer resistance to TNF-related apoptosis-inducing ligand. J Biol Chem 292: 10390-10397, 2017.
63. Zhang L, Yang Z, Trottier J, Barbier O and Wang L: Long noncoding RNA MEG3 induces cholestatic liver injury by interaction with PTBP1 to facilitate shp mRNA decay. Hepatology 65: 604-615, 2017.

64. Volinia S, Visone R, Galasso M, Rossi E and Croce CM: Identification of microRNA activity by targets' reverse EXpression. Bioinformatics 26: 91-97, 2010.

65. Martens-Uzunova ES, Böttcher R, Croce CM, Jenster G, Visakorpi T and Calin GA: Long noncoding RNA in prostate, bladder, and kidney cancer. Eur Urol 65: 1140-1151, 2014.

66. Vallot C, Patrat C, Collier AJ, Huret C, Casanova M, Liyakat Ali TM, Tosolini M, Frydman N, Heard E, Rugg-Gunn PJ and Rougeulle C: XACT noncoding RNA competes with XIST in the control of $\mathrm{X}$ chromosome activity during human early development. Cell Stem Cell 20: 102-111, 2017.

67. Xie VK, Li Z, Yan Y, Jia Z,Zuo X, Ju Z, Wang J, Du J, Xie D, Xie K and Wei D: DNA-Methyltransferase 1 induces dedifferentiation of pancreatic cancer cells through silencing of Kruppel-Like factor 4 expression. Clin Cancer Res 23: 5585-5597, 2017.

68. Zhou DD, Liu XF, Lu CW, Pant OP and Liu XD: Long non-coding RNA PVT1: Emerging biomarker in digestive system cancer. Cell Prolif: Oct 12, 2017 (Epub ahead of print). doi: 10.1111/cpr.12398.

69. Kim K, Jutooru I, Chadalapaka G, Johnson G, Frank J, Burghardt R, Kim S and Safe S: HOTAIR is a negative prognostic factor and exhibits pro-oncogenic activity in pancreatic cancer. Oncogene 32: 1616-1625, 2013

70. Wang J, Zhao H, Fan Z, Li G, Ma Q, Tao Z, Wang R, Feng J and Luo Y: Long noncoding RNA H19 promotes neuroinflammation in ischemic stroke by driving histone deacetylase 1-dependent M1 microglial polarization. Stroke 48: 2211-2221, 2017.

71. Wang G,Pan J,Zhang L, Wei Y and WangC: Long non-coding RNA CRNDE sponges miR-384 to promote proliferation and metastasis of pancreatic cancer cells through upregulating IRS1. Cell Prolif: Sep 21, 2017 (Epub ahead of print). doi: 10.1111/cpr.12389.

72. Pang EJ, Yang R, Fu XB and Liu YF: Overexpression of long non-coding RNA MALAT1 is correlated with clinical progression and unfavorable prognosis in pancreatic cancer. Tumour Biol 36: 2403-2407, 2015

73. Liu L, An X, Li Z, Song Y, Li L, Zuo S, Liu N, Yang G, Wang H, Cheng X, et al: The H19 long noncoding RNA is a novel negative regulator of cardiomyocyte hypertrophy. Cardiovasc Res 111: 56-65, 2016.

74. Heiler S, Wang Z and Zöller M: Pancreatic cancer stem cell markers and exosomes-the incentive push. World J Gastroenterol 22: 5971-6007, 2016.

75. Fuschi P, Maimone B, Gaetano C and Martelli F: Noncoding RNAs in the vascular system response to oxidative stress. Antioxid Redox Signal 30: 992-1010, 2019.

76. Sethi S, Sethi S and Bluth MH: Clinical implication of MicroRNAs in molecular pathology: An update for 2018. Clin Lab Med 38: 237-251, 2018.

77. Vicentini C, Fassan M, D'Angelo E, Corbo V, Silvestris N, Nuovo GJ and Scarpa A: Clinical application of microRNA testing in neuroendocrine tumors of the gastrointestinal tract. Molecules 19: 2458-2468, 2014.

78. Tang YT, Xu XH, Yang XD, Hao J, Cao H, Zhu W, Zhang SY and Cao JP: Role of non-coding RNAs in pancreatic cancer: The bane of the microworld. World J Gastroenterol 20: 9405-9417, 2014

79. Zhang $\mathrm{S}$, Zhu D, Li H,Li H,Feng C and Zhang W: Characterization of circRNA-associated-ceRNA networks in a senescenceaccelerated mouse prone 8 brain. Mol Ther 25: 2053-2061, 2017.

80. Kishikawa T, Otsuka M, Ohno M, Yoshikawa T, Takata A and Koike K: Circulating RNAs as new biomarkers for detecting pancreatic cancer. World J Gastroenterol 21: 8527-8540, 2015.

81. Knupp D and Miura P: CircRNA accumulation: A new hallmark of aging? Mech Ageing Dev 173: 71-79, 2018.

82. Li X, Liu CX, Xue W, Zhang Y, Jiang S, Yin QF, Wei J, Yao RW, Yang L and Chen LL: Coordinated circRNA biogenesis and function with NF90/NF110 in viral infection. Mol Cell 67: 214-227.e7, 2017.

83. Vassella E, Chaudhary AK, Mondal G, Kumar V, Kattel K and Mahato RI: Chemosensitization and inhibition of pancreatic cancer stem cell proliferation by overexpression of microRNA-205. Cancer Lett 402: 1-8, 2017.

84. Yoshida K, Toden S, Ravindranathan P, Han H and Goel A: Curcumin sensitizes pancreatic cancer cells to gemcitabine by attenuating PRC2 subunit EZH2, and the lncRNA PVT1 expression. Carcinogenesis 38: 1036-1046, 2017.

85. Zhang J, Wang P, Wan L, Xu S and Pang D: The emergence of noncoding RNAs as Heracles in autophagy. Autophagy 13: 1004-1024, 2017

This work is licensed under a Creative Commons Attribution-NonCommercial-NoDerivatives 4.0 International (CC BY-NC-ND 4.0) License. 